\title{
Common Fixed Points of Locally Contractive Mappings in Multiplicative Metric Spaces with Application
}

\author{
Mujahid Abbas, ${ }^{1}$ Bashir Ali, ${ }^{2}$ and Yusuf I. Suleiman ${ }^{3}$ \\ ${ }^{1}$ Department of Mathematics and Applied Mathematics, University of Pretoria, Pretoria, South Africa \\ ${ }^{2}$ Department of Mathematics, Bayero University Kano, PMB 3011, Kano, Nigeria \\ ${ }^{3}$ Department of Mathematics, Kano University of Science and Technology, PMB 3042, Wudil, Kano, Nigeria
}

Correspondence should be addressed to Yusuf I. Suleiman; yubram@yahoo.com

Received 12 November 2014; Accepted 26 December 2014

Academic Editor: Onesimo Hernandez-Lerma

Copyright (C) 2015 Mujahid Abbas et al. This is an open access article distributed under the Creative Commons Attribution License, which permits unrestricted use, distribution, and reproduction in any medium, provided the original work is properly cited.

\begin{abstract}
The aim of this paper is to present common fixed point results of quasi-weak commutative mappings on a closed ball in the framework of multiplicative metric spaces. Example is presented to support the result proved herein. We also study sufficient conditions for the existence of a common solution of multiplicative boundary value problem. Our results extend and improve various recent results in the existing literature.
\end{abstract}

\section{Introduction and Preliminaries}

The letters $\mathbb{R}, \mathbb{R}^{+}$, and $\mathbb{N}$ will denote the set of all real numbers, the set of all nonnegative real numbers, and the set of all natural numbers, respectively.

Consistent with $[1,2]$, the following definitions and results will be needed in the sequel.

Definition 1 (see [2]). The multiplicative absolute value function $|\cdot|: \mathbb{R}^{+} \rightarrow \mathbb{R}^{+}$is defined as

$$
|x|= \begin{cases}x, & x \geq 1 ; \\ \frac{1}{x}, & x<1 .\end{cases}
$$

Using above definition of multiplicative absolute value function, we deduce the following proposition.

Proposition 2. For arbitrary $x, y \in \mathbb{R}^{+}$, the following hold:

(1) $|x| \geq 1$;

(2) $1 /|x| \leq x \leq|x|$;

(3) $|1 / x|=|x|$;

(4) $|x| \leq y$ if and only if $1 / y \leq x \leq y$;

(5) $|x \cdot y| \leq|x||y|$.
Bashirov et al. [1] studied the concept of multiplicative calculus and proved the fundamental theorem of multiplicative calculus. Florack and Assen [3] displayed the use of the concept of multiplicative calculus in biomedical image analysis. Bashirov et al. [4] exploit the efficiency of multiplicative calculus over the Newtonian calculus. They demonstrated that the multiplicative differential equations are more suitable than the ordinary differential equations in investigating some problems in various fields. Furthermore, Bashirov et al. [1] illustrated the usefulness of multiplicative calculus with some interesting applications. With the help of multiplicative absolute value function, they defined the multiplicative distance between two nonnegative real numbers as well as between two positive square matrices. This provides the basis for multiplicative metric spaces.

Definition 3 (see [1]). Let $X$ be a nonempty set. A function $d: X^{2} \rightarrow \mathbb{R}^{+}$is said to be a multiplicative metric on $X$ if for any $x, y, z \in X$, the following conditions hold:

$$
\begin{aligned}
& \left(m_{1}\right) d(x, y) \geq 1 \text { and } d(x, y)=1 \text { if and only if } x=y ; \\
& \left(m_{2}\right) d(x, y)=d(y, x) ; \\
& \left(m_{3}\right) d(x, y) \leq d(x, z) \cdot d(z, y) .
\end{aligned}
$$

The pair $(X, d)$ is called a multiplicative metric space. 
Example 4 (see [2]). Let $X=\mathbb{R}_{n}^{+}$be the collection of all $n$-tuples of positive real numbers. Then $d(x, y)=\left|x_{1} / y_{1}\right|$. $\left|x_{2} / y_{2}\right| \cdot \ldots \cdot\left|x_{n} / y_{n}\right|$ defines a multiplicative metric on $X$.

Definition 5 (see [2]). Let $x_{0}$ be an arbitrary point in a multiplicative metric space $X$ and $\epsilon>1$. A multiplicative open ball $B\left(x_{0}, \epsilon\right)$ of radius $\epsilon$ centered at $x_{0}$ is the set

$$
\left\{y \in X: d\left(y, x_{0}\right)<\epsilon\right\} .
$$

A sequence $\left\{x_{n}\right\}$ in multiplicative metric space $X$ is said to be multiplicative convergent to a point $x \in X$ if for any given $\epsilon>1$, there is $N \in \mathbb{N}$ such that $x_{n} \in B(x, \epsilon)$ for all $n \geq N$. If $\left\{x_{n}\right\}$ converges to $x$, we write $x_{n} \rightarrow x$ as $n \rightarrow \infty$. A sequence $\left\{x_{n}\right\}$ in $X$ is multiplicative convergent to $x$ in $X$ if and only if $d\left(x_{n}, x\right) \rightarrow 1$ as $n \rightarrow \infty[2]$.

Definition 6. Let $\left(X, d_{X}\right)$ and $\left(Y, d_{Y}\right)$ be multiplicative metric spaces and $x_{0}$ an arbitrary but fixed point in $X$. A map $f$ : $X \rightarrow Y$ is said to be multiplicative continuous at $x_{0}$ if and only if $x_{n} \rightarrow x_{0}$ in $\left(X, d_{X}\right)$ implies $f\left(x_{n}\right) \rightarrow f\left(x_{0}\right)$ in $\left(Y, d_{Y}\right)$ for every multiplicative convergent sequence $\left\{x_{n}\right\}$ in $X$. That is, given arbitrary $\epsilon>1$, there exists $\delta\left(x_{0}, \epsilon\right)>1$ such that $d_{Y}\left(f x, f x_{0}\right)<\epsilon$ whenever $d_{X}\left(x, x_{0}\right)<\delta$ for $x \in X$.

Example 7. Let $X=C^{*}[a, b]$ be the collection of all realvalued multiplicative continuous functions over $[a, b] \subseteq \mathbb{R}^{+}$. Then $(X, d)$ is a multiplicative metric space with $d$ defined by

$$
d(f, g)=\sup _{x \in[a, b]}\left|\frac{f(x)}{g(x)}\right| \quad \text { for arbitrary } f, g \in X .
$$

For more examples of multiplicative metric spaces, we refer to $[1,2]$.

Definition 8 (see [2]). Let $(X, d)$ be a multiplicative metric space.

(a) A sequence $\left\{x_{n}\right\}$ in $X$ is said to be multiplicative Cauchy sequence if for any $\epsilon>1$, there exists $N \in \mathbb{N}$ such that $d\left(x_{n}, x_{m}\right) \leq \epsilon$ for all $m, n>N$.

(b) A multiplicative metric space $(X, d)$ is said to be complete if every Cauchy sequence $\left\{x_{n}\right\}$ in $X$ is multiplicative convergent to a point $x \in X$.

A sequence $\left\{x_{n}\right\}$ in $X$ is multiplicative Cauchy if and only if $d\left(x_{n}, x_{m}\right) \rightarrow 1$ as $n, m \rightarrow \infty$ [2].

For sake of brevity we skip the proof of the following lemma.

Lemma 9. Let $X=C^{*}[a, b]$ be the collection of all real-valued multiplicative continuous functions over $[a, b] \subseteq \mathbb{R}^{+}$with the metric d defined by

$$
d(f, g)=\sup _{x \in[a, b]}\left|\frac{f(x)}{g(x)}\right|
$$

Then $\left(C^{*}[a, b], d\right)$ is complete.

Definition 10 (see [5]). Let $f, g: X \rightarrow X$ be maps. A point $x \in X$ is called
(1) fixed point of $f$ if $f x=x$;

(2) coincidence point of the pair $\{f, g\}$ if $f x=g x$;

(3) common fixed point of the pair $\{f, g\}$ if $x=f x=g x$.

The sets of all fixed points of $f$, coincidence points of the pair $(f, g)$, and all common fixed points of the pair $(f, g)$ are denoted by $F(f), C(f, g)$, and $F(f, g)$, respectively.

One of the simplest and most useful results in fixed point theory is the Banach-Caccioppoli contraction mapping principle, a powerful tool in analysis for establishing existence and uniqueness of solution of problems in different fields. Over the years, this principle has been generalized in numerous directions in different spaces. These generalizations have been obtained either by extending the domain of the mapping or by considering a more general contractive condition on the mappings.

Recently, Ozavsar and Cervikel [2] generalized the celebrated Banach contraction mapping principle in the setup of multiplicative metric spaces.

Definition 11 (see [2]). Let $X$ be a multiplicative metric space. A mapping $f: X \rightarrow X$ is said to be multiplicative contractive if there exists $\lambda \in[0,1)$ such that

$$
d(f x, f y) \leq(d(x, y))^{\lambda} \quad \forall x, y \in X
$$

Theorem 12 (see [2]). Let $X$ be a complete multiplicative metric space and $f: X \rightarrow X$ a multiplicative contractive mapping. Then $f$ has a unique fixed point.

Definition 13. Let $(X, d)$ be a multiplicative metric space and let $f, g: X \rightarrow X$. The mapping $f$ is said to be $g$ multiplicative contraction if there exists $k \in[0,1)$ such that

$$
d(f x, f y) \leq(d(g x, g y))^{k} \quad \forall x, y \in X .
$$

Definition 14 (see [5]). Let $X$ be a multiplicative metric space and $f, g: X \rightarrow X$. The pair $(f, g)$ is said to be

(a) commutative if $f g x=g f x$ for all $x$ in $X$

(b) weakly commutative if $d(f g x, g f x) \leq d(f x, g x)$ for all $x$ in $X$.

He et al. [6] extended the results in [2] to two pairs of self-mappings satisfying certain commutative conditions on a multiplicative metric space. They actually proved the following result.

Theorem 15. Let $S, T, A$, and $B$ be self-maps of a complete multiplicative metric space $X$ and $(A, S)$ and $(B, T)$ weakly commuting pair with $S X \subset B X, T X \subset A X$, and one of the mappings $S, T, A$, and $B$ is continuous. If

$$
d(S x, T y) \leq(M(x, y))^{\lambda} \quad \text { for any } x, y \in X,
$$

where $M(x, y)=\max \{d(A x, B y), d(A x, S x), d(B y, T y), d(S x$, $B y), d(A x, T y)\}$ for $\lambda \in(0,1 / 2)$ holds. Then $S, T, A$, and $B$ have a unique common fixed point. 
The study of contractive conditions on the entire domain has been at the center of vigorous research activity (see [7] and references therein) and it has a wide range of applications in different areas such as nonlinear and adoptive control systems, parameterize estimation problems, fractal image decoding, computing magnetostatic fields in a nonlinear medium, and convergence of recurrent networks (see, e.g., [8-11]).

If a mapping $f$ does not satisfy a contractive condition on the entire space $X$, a natural question arising in that direction is, whether it is still possible to guarantee the existence of a fixed point. An affirmative answer to this question is provided by (a) the restriction of the domain to the subset $Y$ of $X$, where the mapping $f$ is contractive (b) the suitable choice of a point $x_{0}$ in $X$ which force the Picard sequence to stay within the set $Y$. Recently Azam et al. [12] (see also, [13]) proved a significant result concerning the existence of fixed point of a mapping satisfying contractive conditions on a closed ball of a complete metric space.

The purpose of this paper is to establish the existence and uniqueness of common fixed point of quasi-weak commutative contractive mappings defined on a closed ball in a multiplicative metric space. Our results extend and improve the results of He et al. [6], Arshad et al. [13], and many others.

\section{Main Result}

In this section, we obtain several common fixed point results of mappings on multiplicative closed balls in the framework of multiplicative metric spaces.

We start with the following result.

Theorem 16. Let $S, T, f$, and $g$ be self-maps of a complete multiplicative metric space $X$ and $(f, S)$ and $(g, T)$ weakly commutative with $S X \subset g X, T X \subset f X$, and one of $S, T, f$, and $g$ is continuous. If $S x_{0}=y_{0}$ for some given point $x_{0}$ in $X$ and there exists $\lambda \in(0,1 / 2)$ with $h=\lambda /(1-\lambda)$ such that

$$
d(S x, T y) \leq(M(x, y))^{\lambda} \quad \text { for any } x, y \in \overline{B\left(y_{0}, r\right)}
$$

holds, where $M(x, y)=\max \{d(f x, g y), d(f x, S x), d(g y, T y)$, $d(S x, g y), d(f x, T y)\}$. Then there exists a unique common fixed point of $f, T, S$, and $g$ in $\overline{B\left(y_{0}, r\right)}$ provided that $d\left(y_{0}, T x_{1}\right) \leq r^{(1-h)}$ for some $x_{1}$ in $X$.

Proof. Let $x_{0}$ be a given point in $X$. Since $S X \subset g X$, we can choose a point $x_{1}$ in $X$ such that $S x_{0}=g x_{1}=y_{0}$. Similarly, there exists a point $x_{2} \in X$ such that $T x_{1}=f x_{2}=y_{1}$. Indeed, it follows from the assumption that $T X \subset f X$. Thus we can construct sequences $\left\{x_{n}\right\}$ and $\left\{y_{n}\right\}$ in $X$ such that

$$
\begin{array}{r}
y_{2 n}=S x_{2 n}=g x_{2 n+1}, \quad y_{2 n+1}=T x_{2 n+1}=f x_{2 n+2} \\
\text { for } n=0,1,2, \ldots
\end{array}
$$

Now we show that $\left\{y_{n}\right\}$ is a sequence in $\overline{B\left(y_{0}, r\right)}$. Note that $d\left(y_{0}, y_{1}\right)=d\left(y_{0}, T x_{1}\right) \leq r^{(1-h)}<r$. Hence $y_{1} \in \overline{B\left(y_{0}, r\right)}$.
Assume $y_{2}, y_{3}, \ldots, y_{j} \in \overline{B\left(y_{0}, r\right)}$ for some $j \in \mathbb{N}$. Then, if $j=2 k$, it follows from (8) that

$$
\begin{gathered}
d\left(y_{2 k}, y_{2 k+1}\right) \\
=d\left(S x_{2 k}, T x_{2 k+1}\right) \\
\leq\left(\operatorname { m a x } \left\{d\left(f x_{2 k}, g x_{2 k+1}\right), d\left(f x_{2 k}, S x_{2 k}\right)\right.\right. \\
d\left(g x_{2 k+1}, T x_{2 k+1}\right), d\left(S x_{2 k}, g x_{2 k+1}\right) \\
\left.\left.d\left(f x_{2 k}, T x_{2 k+1}\right)\right\}\right)^{\lambda} \\
\leq\left(\operatorname { m a x } \left\{d\left(y_{2 k-1}, y_{2 k}\right), d\left(y_{2 k-1}, y_{2 k}\right), d\left(y_{2 k}, y_{2 k+1}\right),\right.\right. \\
\left.\left.\quad d\left(y_{2 k}, y_{2 k}\right), d\left(y_{2 k-1}, y_{2 k+1}\right)\right\}\right)^{\lambda} \\
\left.\left.\quad d\left(y_{2 k-1}, y_{2 k+1}\right)\right\}\right)^{\lambda} \\
\leq\left(\operatorname { m a x } \left\{d\left(y_{2 k-1}, y_{2 k}\right), d\left(y_{2 k}, y_{2 k+1}\right), 1\right.\right. \\
\left.\left.d\left(y_{2 k-1}, y_{2 k}\right) \cdot d\left(y_{2 k}, y_{2 k+1}\right)\right\}\right)^{\lambda} \\
=d\left(y_{2 k-1}, y_{2 k}\right)^{\lambda} \cdot d\left(y_{2 k}, y_{2 k+1}\right)^{\lambda} .
\end{gathered}
$$

Thus $d\left(y_{2 k}, y_{2 k+1}\right) \leq\left(d\left(y_{2 k-1}, y_{2 k}\right)\right)^{h}$ for all $k \in \mathbb{N}$, where $h=$ $\lambda /(1-\lambda)$.

Similarly, $j=2 k+1$, and we obtain

$$
d\left(y_{2 k+1}, y_{2 k+2}\right) \leq d\left(y_{2 k}, y_{2 k+1}\right)^{h} .
$$

Hence

$$
d\left(y_{k}, y_{k+1}\right) \leq d\left(y_{k-1}, y_{k}\right)^{h} \quad \text { for } k \in \mathbb{N} \text {. }
$$

Therefore

$$
\begin{aligned}
d\left(y_{k}, y_{k+1}\right) & \leq d\left(y_{k-1}, y_{k}\right)^{h} \leq d\left(y_{k-2}, y_{k-1}\right)^{h^{2}} \\
& \leq \cdots \leq d\left(y_{0}, y_{1}\right)^{h^{k}}
\end{aligned}
$$

for all $k \in \mathbb{N}$. Now

$$
\begin{gathered}
d\left(y_{0}, y_{k+1}\right) \leq \\
d\left(y_{0}, y_{1}\right) \cdot d\left(y_{1}, y_{2}\right) \cdot d\left(y_{2}, y_{3}\right) \\
\cdot \ldots \cdot d\left(y_{k}, y_{k+1}\right) .
\end{gathered}
$$

Thus

$$
\begin{aligned}
d\left(y_{0}, y_{k+1}\right) \leq & d\left(y_{0}, y_{1}\right) \cdot d\left(y_{0}, y_{1}\right)^{h} \cdot d\left(y_{0}, y_{1}\right)^{h^{2}} \\
& \cdot \ldots \cdot d\left(y_{0}, y_{1}\right)^{h^{k}} \\
\leq & d\left(y_{0}, y_{1}\right)^{\left(h^{0}+h^{1}+h^{2}+\cdots+h^{k}\right)} \\
\leq & d\left(y_{0}, y_{1}\right)^{\left(1-h^{k+1}\right) /(1-h)} .
\end{aligned}
$$

Since $y_{1} \in \overline{B\left(y_{0}, r\right)}$, we have that $d\left(y_{0}, y_{k+1}\right) \leq$ $\left(r^{(1-h)}\right)^{\left(\left(1-h^{k+1}\right) /(1-h)\right)} \leq r^{\left(1-h^{k+1}\right)} \leq r$ for all $k \in \mathbb{N}$. This 
implies $y_{k+1} \in \overline{B\left(y_{0}, r\right)}$. By induction on $n$, we conclude that $\left\{y_{n}\right\} \in \overline{B\left(y_{0}, r\right)}$ for all $n \in \mathbb{N}$. We claim that the sequence $\left\{y_{n}\right\}$ satisfies the multiplicative Cauchy criterion for convergence in $\left(\overline{B\left(y_{0}, r\right)}, d\right)$. To see this let $m, n \in \mathbb{N}$ be such that $m>n$; then

$$
\begin{aligned}
d\left(y_{n}, y_{m}\right) & \leq d\left(y_{n}, y_{n+1}\right) \cdot d\left(y_{n+1}, y_{n+2}\right) \cdot \ldots \cdot d\left(y_{m-1}, y_{m}\right) \\
& \leq d\left(y_{0}, y_{1}\right)^{h^{n}} \cdot d\left(y_{0}, y_{1}\right)^{h^{n+1}} \cdot \ldots \cdot d\left(y_{0}, y_{1}\right)^{h^{m-1}} \\
& \leq d\left(y_{0}, y_{1}\right)^{\left(h^{n}+h^{n+1}+\cdots+h^{m-1}\right)} \\
& \leq d\left(y_{0}, y_{1}\right)^{\left(h^{n}+h^{n+1}+\cdots\right)} \\
& \leq d\left(y_{0}, y_{1}\right)^{\left(h^{n} /(1-h)\right)} .
\end{aligned}
$$

Consequently $d\left(y_{m}, y_{n}\right) \rightarrow 1$ as $n, m \rightarrow \infty$. Hence the sequence $\left\{y_{n}\right\}$ is a multiplicative Cauchy sequence.

As $X$ is complete so is $\overline{B\left(y_{0}, r\right)}$. Hence $\left\{y_{n}\right\}$ has a limit, say $u$, in $\overline{B\left(y_{0}, r\right)}$.

The fact that $\left\{S x_{2 n}\right\}=\left\{g x_{2 n+1}\right\}=\left\{y_{2 n}\right\}$ and $\left\{T x_{2 n+1}\right\}=\left\{f x_{2 n+2}\right\}=\left\{y_{2 n+1}\right\}$ are subsequences of $\left\{y_{n}\right\}$ makes $\lim _{n \rightarrow \infty} S x_{2 n}=\lim _{n \rightarrow \infty} g x_{2 n+1}=\lim _{n \rightarrow \infty} T x_{2 n+1}=$ $\lim _{n \rightarrow \infty} f x_{2 n+2}=u$.

Suppose $f$ is continuous; then

$$
\lim _{n \rightarrow \infty} f\left(S x_{2 n}\right)=f\left(\lim _{n \rightarrow \infty} S x_{2 n}\right)=f\left(\lim _{n \rightarrow \infty} f x_{2 n+2}\right)=f(u) .
$$

By weak commutativity of a pair $\{f, S\}$, we have

$$
d\left(f\left(S x_{2 n}\right), S\left(f x_{2 n}\right)\right) \leq d\left(f x_{2 n}, S x_{2 n}\right) .
$$

Taking limit as $n \rightarrow \infty$ on both sides of (18), we get

$$
d\left(f(u), \lim _{n \rightarrow \infty} S\left(f x_{2 n}\right)\right) \leq d(u, u)
$$

which further implies that $\lim _{n \rightarrow \infty} S\left(f x_{2 n}\right)=f(u)$. Now by condition (8), we have

$$
\begin{gathered}
d\left(S\left(f x_{2 n}\right), T x_{2 n+1}\right) \\
\leq\left(\operatorname { m a x } \left\{d\left(f^{2} x_{2 n}, g x_{2 n+1}\right), d\left(f^{2} x_{2 n}, S f x_{2 n}\right),\right.\right. \\
d\left(g x_{2 n+1}, T x_{2 n+1}\right), d\left(S f x_{2 n}, g x_{2 n+1}\right), \\
\left.\left.d\left(f^{2} x_{2 n}, T x_{2 n+1}\right)\right\}\right)^{\lambda} .
\end{gathered}
$$

Taking limit as $n \rightarrow \infty$ on both sides of (20), we obtain

$$
\begin{gathered}
d(f u, u) \leq(\max \{d(f u, u), d(f u, f u), d(u, u), \\
d(f u, u), d(f u, u)\})^{\lambda} .
\end{gathered}
$$

That is, $d(f u, u) \leq d(f u, u)^{\lambda}$. Hence $d(f u, u)=1$ and $u$ is a fixed point of $f$ in $\overline{B\left(y_{0}, r\right)}$. In similar way, by condition (8) we have

$$
\begin{gathered}
d\left(S(u), T x_{2 n+1}\right) \\
\leq\left(\operatorname { m a x } \left\{d\left(f u, g x_{2 n+1}\right), d(f u, S u), d\left(g x_{2 n+1}, T x_{2 n+1}\right)\right.\right. \\
\left.\left.d\left(S u, g x_{2 n+1}\right), d\left(f u, T x_{2 n+1}\right)\right\}\right)^{\lambda}
\end{gathered}
$$

Taking limit as $n \rightarrow \infty$ on both sides of (22), we get

$$
\begin{gathered}
d(S u, u) \leq(\max \{d(f u, u), d(u, S u), d(u, u), \\
d(S u, u), d(u, u)\})^{\lambda}
\end{gathered}
$$

Hence $d(S u, u)=1$ and $u$ is a fixed point of $S$ in $\overline{B\left(y_{0}, r\right)}$.

Because of the fact that $u=S(u) \in S\left(\overline{B\left(y_{0}, r\right)}\right) \subseteq$ $g\left(\overline{B\left(y_{0}, r\right)}\right)$, let $u^{*}$ in $\left(\overline{B\left(y_{0}, r\right)}\right)$ be such that $u=g\left(u^{*}\right)$. So it follows from (8) that

$$
\begin{aligned}
d\left(u, T u^{*}\right) & =d\left(S(u), T u^{*}\right) \\
\leq & \left(\operatorname { m a x } \left\{d\left(f u, g u^{*}\right), d(f u, S u), d\left(g u^{*}, T u^{*}\right),\right.\right. \\
& \left.\left.d\left(S u, g u^{*}\right), d\left(f u, T u^{*}\right)\right\}\right)^{\lambda}
\end{aligned}
$$

which implies $d\left(u, T u^{*}\right)=1$ and $T u^{*}=u$. Since the pair $\{f, T\}$ is weakly commutative from our assumptions, thus

$d(g u, T u)=d\left(g T u^{*}, T g u^{*}\right) \leq d\left(g u^{*}, T u^{*}\right)=d(u, u)=1$.

Hence $g u=T u$. By (8), we obtain

$$
\begin{gathered}
d(u, T u)=d(S(u), T u) \\
\leq(\max \{d(f u, g u), d(f u, S u), d(g u, T u), \\
\quad d(S u, g u), d(f u, T u)\})^{\lambda},
\end{gathered}
$$

which implies $u=T(u)$. Hence $u$ is a common fixed point of $f, g, S$, and $T$ in $\overline{B\left(y_{0}, r\right)}$. If $g$ is continuous, then following arguments similar to those given above, we obtain that $u=$ $S(u)=f(u)=T(u)=g(u)$. Now suppose that $S$ is continuous. Thus

$$
\lim _{n \rightarrow \infty} S\left(f x_{2 n}\right)=S\left(\lim _{n \rightarrow \infty} S x_{2 n}\right)=S(u) .
$$

As the pair $\{f, S\}$ is weakly commuting, we have

$$
d\left(f\left(S x_{2 n}\right), S\left(f x_{2 n}\right)\right) \leq d\left(f x_{2 n}, S x_{2 n}\right) .
$$

Taking limit as $n \rightarrow \infty$ on both sides of (28), we have $d\left(\lim _{n \rightarrow \infty} f\left(S x_{2 n}\right), S u\right) \leq d(u, u)=1$ and $\lim _{n \rightarrow \infty} f\left(S x_{2 n}\right)=$ $S(u)$. 
By contractive condition (8) we get

$$
\begin{gathered}
d\left(S\left(S x_{2 n}\right), T x_{2 n+1}\right) \\
\leq\left(\operatorname { m a x } \left\{d\left(f S x_{2 n}, g x_{2 n+1}\right), d\left(f S x_{2 n}, f S x_{2 n}\right),\right.\right. \\
d\left(g x_{2 n+1}, T x_{2 n+1}\right), d\left(S S x_{2 n}, g x_{2 n+1}\right), \\
\left.\left.d\left(f S x_{2 n}, T x_{2 n+1}\right)\right\}\right)^{\lambda} .
\end{gathered}
$$

Taking limit as $n \rightarrow \infty$ on both sides of (29) implies that $d(S u, u) \leq d(S u, u)^{\lambda}$.

Hence $d(S u, u)=1$ and $u$ is a fixed point of $S$ in $\overline{B\left(y_{0}, r\right)}$.

Since $u=S(u) \in S\left(\overline{B\left(y_{0}, r\right)}\right) \subseteq g\left(\overline{B\left(y_{0}, r\right)}\right)$, let $u^{*}$ in $\left(\overline{B\left(y_{0}, r\right)}\right)$ be such that $u=g\left(u^{*}\right)$. It follows from condition (8) again that

$$
\begin{aligned}
& d\left(S S x_{2 n}, T u^{*}\right) \\
& \leq\left(\operatorname { m a x } \left\{d\left(f S x_{2 n}, g u^{*}\right), d\left(f S x_{2 n}, S S x_{2 n}\right),\right.\right. \\
& \left.\left.\quad d\left(g u^{*}, T u^{*}\right), d\left(S S x_{2 n}, g u^{*}\right), d\left(f S x_{2 n}, T u^{*}\right)\right\}\right)^{\lambda} .
\end{aligned}
$$

Taking limit as $n \rightarrow \infty$ on both sides of (30) implies that $d\left(u, T u^{*}\right) \leq d\left(u, T u^{*}\right)^{\lambda}$. Thus $T u^{*}=u$. Since the pair $\{T, g\}$ is weakly commutative from our hypothesis, then

$$
d(T u, g u)=d\left(T g u^{*}, g T u^{*}\right) \leq d\left(T u^{*}, g u^{*}\right)=d(u, u)=1
$$

which implies that $g u=T u$. From (8), we have

$$
\begin{gathered}
d\left(S x_{2 n}, T u\right) \\
\leq\left(\operatorname { m a x } \left\{d\left(f x_{2 n}, g u\right), d\left(f x_{2 n}, S x_{2 n}\right), d(g u, T u),\right.\right. \\
\left.\left.d\left(S x_{2 n}, g u\right), d\left(f x_{2 n}, T u\right)\right\}\right)^{\lambda} .
\end{gathered}
$$

Taking limit as $n \rightarrow \infty$ on both sides of (32) gives $d(u, T u) \leq$ $d(u, T u)^{\lambda}$ and $u=T(u)$. However $u=T(u) \in T\left(\overline{B\left(y_{0}, r\right)}\right) \subseteq$ $f\left(\overline{B\left(y_{0}, r\right)}\right)$, so let $v \in\left(\overline{B\left(y_{0}, r\right)}\right)$ be such that $u=f(v)$. It follows from (8) also that

$$
\begin{gathered}
d(S v, u)=d(S v, T u) \\
\leq(\max \{d(f v, g u), d(f v, S v), d(g u, T u), \\
d(S v, g u), d(f v, T u)\})^{\lambda},
\end{gathered}
$$

which implies that $d(S(v), u) \leq d(S(v), u)^{\lambda}$. Hence $S(v)=u$. Since $S$ and $f$ are weakly commutative,

$$
d(f u, S u)=d(f S v, S f v) \leq d(f v, S v)=d(u, u)=1
$$

gives $f(u)=S(u)$. Applying condition (8), we obtain

$$
\begin{gathered}
d(S u, u)=d(S u, T u) \\
\leq(\max \{d(f u, g u), d(f u, S u), d(g u, T u), \\
d(S u, g u), d(f u, T u)\})^{\lambda} \\
=(\max \{d(S u, u), d(S u, S u), d(g u, g u), \\
d(S u, u), d(S u, u)\})^{\lambda}
\end{gathered}
$$

which implies that $u=S(u)$. Hence $u$ is a common fixed point of $f, S, T$, and $g$ in $\overline{B\left(y_{0}, r\right)}$. If $T$ is continuous, then using arguments similar to those given above, the result follows.

We proceed to show the uniqueness of the common fixed point of the maps $f, T, S$, and $g$. So, let $z \in \overline{B\left(y_{0}, r\right)}$ be another common fixed point of $f, T, S$, and $g$. By (8), we have

$$
\begin{gathered}
d(u, z)=d(S u, T z) \\
\leq(\max \{d(f u, g z), d(f u, S u), d(g z, T z), \\
d(S u, g z), d(f u, T z)\})^{\lambda} .
\end{gathered}
$$

That is, $d(u, z) \leq d(u, z)^{\lambda}$. Hence $u=z$ and this implies that the common fixed point of $f, T, S$, and $g$ is unique.

The following result generalizes Theorem 12 . We obtain a common fixed point result in the setup of multiplicative metric spaces without the assumption of continuity and weak commutativity.

Theorem 17. Let $f$ and $g$ be two maps on a complete multiplicative metric space $X$ and $x_{0}$ an arbitrary point in $X$. Suppose that there exists $\lambda$ in $[0,1)$ such that

$$
d(f x, g y) \leq d(x, y)^{\lambda}
$$

for any $x, y \in \overline{B\left(x_{0}, r\right)}$ and $d\left(x_{0}, f\left(x_{0}\right)\right) \leq r^{(1-\lambda)}$ is satisfied. Then, there exists a unique common fixed point of $f$ and $g$ in $\overline{B\left(x_{0}, r\right)}$.

Proof. Let $x_{0}$ be a given point in $X$. Define a sequence $\left\{x_{n}\right\}$ in $X$ such that $x_{2 n+1}=f\left(x_{2 n}\right)$ and $x_{2 n+2}=g\left(x_{2 n+1}\right)$ for all $n \geq 0$. We show that $x_{n} \in \overline{B\left(x_{0}, r\right)}$ for all $n \in \mathbb{N}$. Note that

$$
d\left(x_{0}, x_{1}\right)=d\left(x_{0}, f x_{0}\right) \leq r^{(1-\lambda)} \leq r .
$$

This implies that $x_{1} \in \overline{B\left(x_{0}, r\right)}$. Let $x_{2}, x_{3}, \ldots, x_{j} \in \overline{B\left(x_{0}, r\right)}$ for some $j \in \mathbb{N}$. Clearly, if $j=2 k+1$, then

$$
\begin{aligned}
d\left(x_{2 k+1}, x_{2 k+2}\right) & =d\left(f x_{2 k}, g x_{2 k+1}\right) \\
& \leq d\left(x_{2 k}, x_{2 k+1}\right)^{\lambda} \\
& \leq d\left(x_{2 k-1}, x_{2 k}\right)^{\lambda^{2}} \\
& \vdots \\
& \leq d\left(x_{0}, x_{1}\right)^{\lambda^{2 k+1}} .
\end{aligned}
$$

Similarly, if $j=2 k+2$, then

$$
d\left(x_{2 k+2}, x_{2 k+3}\right) \leq d\left(x_{0}, x_{1}\right)^{\lambda^{2 k+2}} .
$$

Hence for any $k$ in $\mathbb{N}$, we have $d\left(x_{k}, x_{k+1}\right) \leq d\left(x_{0}, x_{1}\right)^{\lambda^{k}}$. Now

$$
\begin{gathered}
d\left(x_{0}, x_{k+1}\right) \leq d\left(x_{0}, x_{1}\right) \cdot d\left(x_{1}, x_{2}\right) \cdot d\left(x_{2}, x_{3}\right) \\
\cdot \ldots \cdot d\left(x_{k}, x_{k+1}\right)
\end{gathered}
$$


implies that

$$
\begin{gathered}
d\left(x_{0}, x_{k+1}\right) \leq d\left(x_{0}, x_{1}\right) \cdot d\left(x_{0}, x_{1}\right)^{\lambda} \cdot d\left(x_{0}, x_{1}\right)^{\lambda^{2}} \\
\cdot \ldots \cdot d\left(x_{0}, x_{1}\right)^{\lambda^{k}}
\end{gathered}
$$

Thus we have

$$
\begin{aligned}
d\left(x_{0}, x_{k+1}\right) \leq & d\left(x_{0}, x_{1}\right) \cdot d\left(x_{0}, x_{1}\right)^{\lambda} \cdot d\left(x_{0}, x_{1}\right)^{\lambda^{2}} \\
& \cdot \ldots \cdot d\left(x_{0}, x_{1}\right)^{\lambda^{k}} \\
\leq & d\left(x_{0}, x_{1}\right)^{\left(\lambda^{0}+\lambda^{1}+\lambda^{2}+\cdots+\lambda^{k}\right)} \\
\leq & d\left(x_{0}, x_{1}\right)^{\left(1-\lambda^{k+1}\right) /(1-\lambda)} .
\end{aligned}
$$

Since $x_{1} \in \overline{B\left(x_{0}, r\right)}$, then $d\left(x_{0}, x_{k+1}\right) \leq\left(r^{(1-\lambda)}\right)^{\left(\left(1-\lambda^{k+1}\right) /(1-\lambda)\right)} \leq$ $r^{\left(1-\lambda^{k+1}\right)} \leq r$ for all $k \in \mathbb{N}$.

Hence $x_{k+1} \in \overline{B\left(x_{0}, r\right)}$. By induction on $n$, we conclude that $\left\{x_{n}\right\} \in \overline{B\left(x_{0}, r\right)}$ for all $n \in \mathbb{N}$. Now we show that $\left\{x_{n}\right\}$ is Cauchy in $\overline{B\left(y_{0}, r\right)}$. Therefore for each $m, n \in \mathbb{N}$ such that $m>n$,

$$
\begin{aligned}
d\left(x_{n}, x_{m}\right) & \leq d\left(x_{n}, x_{n+1}\right) \cdot d\left(x_{n+1}, x_{n+2}\right) \cdot \ldots \cdot d\left(x_{m-1}, x_{m}\right) \\
& \leq d\left(x_{0}, x_{1}\right)^{\lambda^{n}} \cdot d\left(x_{0}, x_{1}\right)^{\lambda^{n+1}} \cdot \ldots \cdot d\left(x_{0}, x_{1}\right)^{\lambda^{m-1}} \\
& \leq d\left(x_{0}, x_{1}\right)^{\left(\lambda^{n}+\lambda^{n+1}+\cdots+\lambda^{m-1}\right)} \\
& \leq d\left(x_{0}, x_{1}\right)^{\left(\lambda^{n}+\lambda^{n+1}+\cdots\right)} \\
& \leq d\left(x_{0}, x_{1}\right)^{\left(\lambda^{n} /(1-\lambda)\right)} .
\end{aligned}
$$

Note that as $n, m \rightarrow \infty$ we get $d\left(x_{m}, x_{n}\right) \rightarrow 1$. Hence $\left\{x_{n}\right\}$ is a multiplicative Cauchy sequence. By the completeness of $X$, it follows that $\lim _{n \rightarrow \infty} x_{n}=u$ for a point $u \in \overline{B\left(x_{0}, r\right)}$. Also, we have

$$
d\left(x_{2 n+1}, g u\right)=d\left(f\left(x_{2 n}\right), g u\right) \leq d\left(x_{2 n}, u\right)^{\lambda}
$$

which on taking limit as $n$ tends to infinity gives

$$
d(u, g u) \leq d(u, u)^{\lambda}
$$

Thus, $u$ is a fixed point of $g$. In a similar manner, we can observe that $u$ is a fixed point of $f$. Thus $f$ and $g$ have a common fixed point in $\overline{B\left(x_{0}, r\right)}$. Note also that $u$ is a unique common fixed point of $f$ and $g$ in $\overline{B\left(x_{0}, r\right)}$. Indeed, if $z$ is another fixed point of $f$ and $g$, then $d(u, z)=d(f u, g z) \leq$ $d(u, z)^{\lambda}$ which implies that $u=z$.

Corollary 18. Let $f$ and $g$ be two maps on a complete multiplicative metric space $(X, d)$. Suppose that there exists $\lambda$ in $[0,1)$ such that

$$
d(f x, g y) \leq d(x, y)^{\lambda} \quad \text { for any } x, y \in X .
$$

Then, there exists a unique common fixed point of $f$ and $g$ in $X$.
Proof. Put $X=\overline{B\left(x_{0}, r\right)}$ in Theorem 17.

Example 19. Let $X=\mathbb{R}$ and $d: \mathbb{R}^{2} \rightarrow \mathbb{R}^{+}$be a multiplicative metric defined by $d(x, y)=e^{|x-y|}$. Note that $(\mathbb{R}, d)$ is a complete multiplicative metric space. Define mappings $f, g, S$ and $T: \mathbb{R} \rightarrow \mathbb{R}$ by

$$
f(x)=2 x, \quad S(x)=x, \quad T(x)=\frac{1}{2} x, \quad g(x)=3 x .
$$

Obviously, maps are continuous, $(f, S)$ and $(T, g)$ are weak commutative with $S(\mathbb{R}) \subset g(\mathbb{R})$, and $T(\mathbb{R}) \subset f(\mathbb{R})$. Choose $x_{0}=2 / 7$; then there exists $x_{1} \in \overline{B(2 / 7, r)}$ such that $S(2 / 7)=$ $g\left(x_{1}\right)=y_{0}=2 / 7$ and $y_{0}=g\left(x_{1}\right)=3 x_{1}=2 / 7$ gives $x_{1}=2 / 21$. Also, $T\left(x_{1}\right)=T(2 / 21)=1 / 21=y_{1}$. Thus

$$
d\left(y_{0}, T\left(x_{1}\right)\right)=e^{(5 / 21)} .
$$

Moreover, for $r=4$ and $\lambda=5 / 21 \in(0,1 / 2)$ with $h=5 / 16 \epsilon$ $[0,1)$ we have

$$
r^{(1-h)}=4^{(11 / 16)}
$$

and so $d\left(y_{0}, T x_{1}\right) \leq r^{(1-h)}$ holds. Note also that

$$
\begin{aligned}
& e^{|x-(1 / 2) y|} \\
& \quad \leq\left(\max \left\{e^{|3 x-2 y|}, e^{|2 x|}, e^{|(3 / 2) y|}, e^{|2 y-x|}, e^{|3 x-(1 / 2) y|}\right\}\right)^{\lambda} .
\end{aligned}
$$

That is,

$$
\begin{gathered}
\left|x-\frac{1}{2} y\right| \leq\left(\operatorname { m a x } \left\{|3 x-2 y| \lambda,|2 x| \lambda,\left|\frac{3}{2} y\right| \lambda,|2 y-x| \lambda,\right.\right. \\
\left.\left.\left|3 x-\frac{1}{2} y\right| \lambda\right\}\right) .
\end{gathered}
$$

Thus all conditions of Theorem 16 are satisfied. Moreover $x=0$ is the unique common fixed point of $f, T, S$, and $g$ in $\overline{B(2 / 7,4)}$.

\section{Application to Multiplicative Boundary Value Problems}

Consider a system of two multiplicative differential equations

$$
\begin{array}{r}
u^{*}(t)=f_{1}(t, u(t)), \quad v^{*}(t)=f_{2}(t, v(t)), \\
\text { with } u(1)=v(1)=u_{0} \text { (say), }
\end{array}
$$

where $t \in[1, T] \subseteq \mathbb{R}^{+}$for sufficiently small $T>1$ and $f_{1}$ and $f_{2}$ are multiplicative continuous functions defined from $[1, T] \times C^{*}[1, T]$ to $\mathbb{R}^{+}$, with $u$ and $v$ in $C^{*}[1, T]$. It is easy to see that solution of problem (53) is equivalently a solution of the following multiplicative integral equations

$$
\begin{aligned}
& u(t)=u_{0} \cdot \int_{1}^{* t} f_{1}(s, u(s))^{d s} \quad \text { for any } s \in[1, T], \\
& v(t)=u_{0} \cdot \int_{1}^{* t} f_{2}(s, v(s))^{d s} \quad \text { for any } s \in[1, T] .
\end{aligned}
$$


Suppose $f_{1}$ and $f_{2}$ satisfy the following multiplicative Lipschitz type condition with respect to second coordinate. That is

$$
\left|\frac{f_{1}(t, u(t))}{f_{2}(t, v(t))}\right| \leq \lambda^{(|u(t) / v(t)|)}
$$

for some constant $\lambda \geq 1$ and $(t, u),(t, v) \in[1, T] \times C^{*}[1, T]$. Let $t_{0} \in[1, T]$; then there exists a unique solution of (54) on some closed interval $\left|t-t_{0}\right| \leq K$, for $K>0$ sufficiently small such that $K^{\lambda}<1$.

Proof. Define $f$ and $g: C^{*}[1, T] \rightarrow C^{*}[1, T]$ as $f u(t)=$ $u_{0} \cdot \int_{t_{0}}^{* t} f_{1}(s, u(s))^{d s}$ and $g v(t)=u_{0} \cdot \int_{t_{0}}^{* t} f_{2}(s, v(s))^{d s}$. Assume $x$ and $y$ are arbitrary members of $C^{*}[1, T]$; then

$$
\begin{aligned}
& d(f x, g y) \\
& =\sup _{t \in[1, T]}\left|\frac{\int_{t_{0}}^{* t} f_{1}(s, x(s))^{d s}}{\int_{t_{0}}^{* t} f_{2}(s, y(s))^{d s}}\right|=\sup _{t \in[1, T]}\left(\left|\int_{t_{0}}^{* t} \frac{f_{1}(s, x(s))}{f_{2}(s, y(s))}\right|\right)^{d s} \\
& \leq \sup _{t \in[1, T]}\left(\int_{t_{0}}^{* t}\left|\frac{f_{1}(s, x(s))}{f_{2}(s, y(s))}\right|\right)^{d s} \leq \sup _{t \in[1, T]} \int_{t_{0}}^{* t}\left(\lambda^{|x(s) / y(s)|}\right)^{d s} \\
& \leq \sup _{t \in[1, T]} \int_{t_{0}}^{* t}\left(\lambda^{\sup _{s \in[1, T]}|x(s) / y(s)|}\right)^{d s}=\sup _{t \in[1, T]} \int_{t_{0}}^{* t}\left(\lambda^{d(x, y)}\right)^{d s} \\
& \quad=\sup _{t \in[1, T]}\left(\int_{t_{0}}^{* t} 1^{d s}\right)^{\lambda^{d(x, y)}}=\sup _{t \in[1, T]}\left(\left|t-t_{0}\right|\right)^{\lambda^{d(x, y)}} \\
& \quad \leq\left(K^{\lambda}\right)^{d(x, y)} \leq d(x, y)^{K^{\lambda}} \quad \text { since } K^{\lambda} \in[0,1) .
\end{aligned}
$$

This implies that $f$ and $g$ satisfy all the hypothesis of Corollary 18; we conclude that $f$ and $g$ admit a unique common fixed point $u \in C^{*}[1, T]$ which is clearly the (unique) common solution to the multiplicative integral equations (54) and hence to (53).

\section{Conflict of Interests}

The authors declare that they have no competing interests.

\section{References}

[1] A. E. Bashirov, E. M. Karplnara, and A. Ozyaplcl, "Multiplicative calculus and its applications," Journal of Mathematical Analysis and Applications, vol. 337, pp. 36-48, 2008.

[2] M. Ozavsar and A. C. Cervikel, "Fixed points of multiplicative contraction mappings on multiplicative metric spaces," http://arxiv.org/abs/1205.5131vl.

[3] L. Florack and H. V. Assen, "Multiplicative calculus in biomedical image analysis," Journal of Mathematical Imaging and Vision, vol. 42, no. 1, pp. 64-75, 2012.

[4] A. E. Bashirov, E. Misirli, Y. Tandogdu, and A. Ozyapici, "On modeling with multiplicative differential equations," Applied Mathematics-A Journal of Chinese Universities, vol. 26, pp. 425-438, 2011.
[5] S. Sessa, "On a weak commutativity condition of mappings in fixed point considerations," Institut Mathématique, vol. 32, pp. 149-153, 1982.

[6] X. He, M. Song, and D. Chen, "Common fixed points for weak commutative mappings on a multiplicative metric space," Fixed Point Theory and Applications, vol. 2014, article 48, 2014.

[7] H. Aydi, "Some fixed point results in ordered partial metric spaces," Journal of Nonlinear Science and Its Applications, vol. 4, no. 3, pp. 210-217, 2011.

[8] K. Leibovic, "The principle of contration mapping in nonlinear and adoptive controle systems," IEEE Transactions on Automatic Control, vol. 9, pp. 393-398, 1964.

[9] G. A. Medrano-Cerda, "A fixed point formulation to parameter estimation problems," in Proceedings of the 26th IEEE Conference on Decision and Control., pp. 1468-1476, 1987.

[10] J. E. Steck, "Convergence of recurrent networks as contraction mappings," Neural Networks, vol. 3, pp. 7-11, 1992.

[11] Y.-M. He and H.-J. Wang, "Fractal image decoding based on extended fixed-point theorem," in Proceedings of the International Conference on Machine Learning and Cybernetics, pp. 4160-4163, Dalian, China, August 2006.

[12] A. Azam, S. Hussain, and M. Arshad, "Common fixed points of Chatterjea type fuzzy mappings on closed balls," Neural Computing and Applications, vol. 21, no. 1, pp. 313-317, 2012.

[13] M. Arshad, A. Shoaib, and I. Beg, "Fixed point of a pair of contractive dominated mappings on a closed ball in an ordered dislocated metric space," Fixed Point Theory and Applications, vol. 2013, article 115, 2013. 


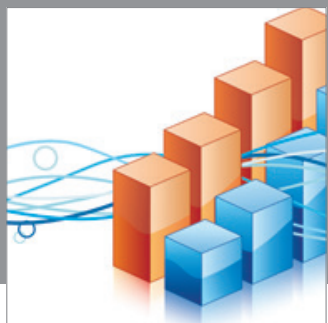

Advances in

Operations Research

mansans

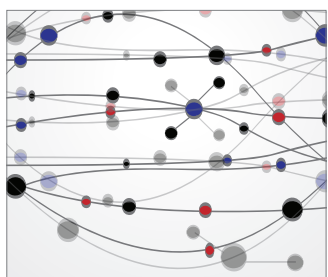

The Scientific World Journal
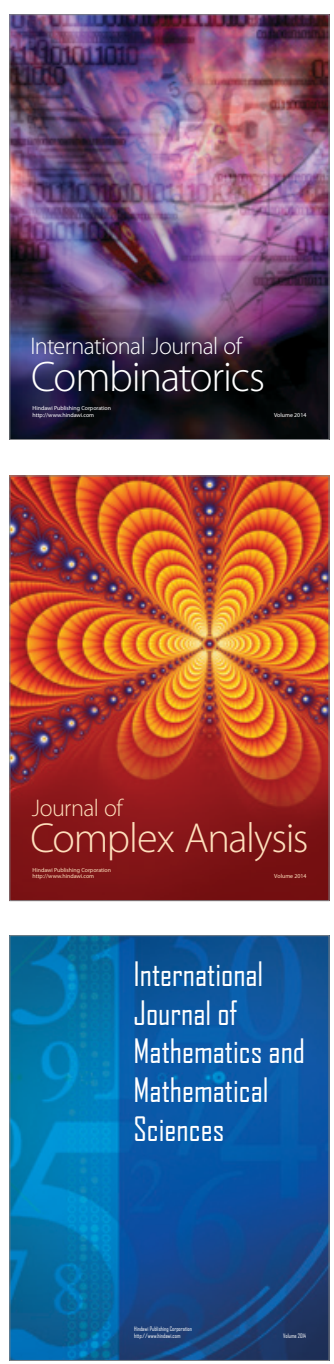
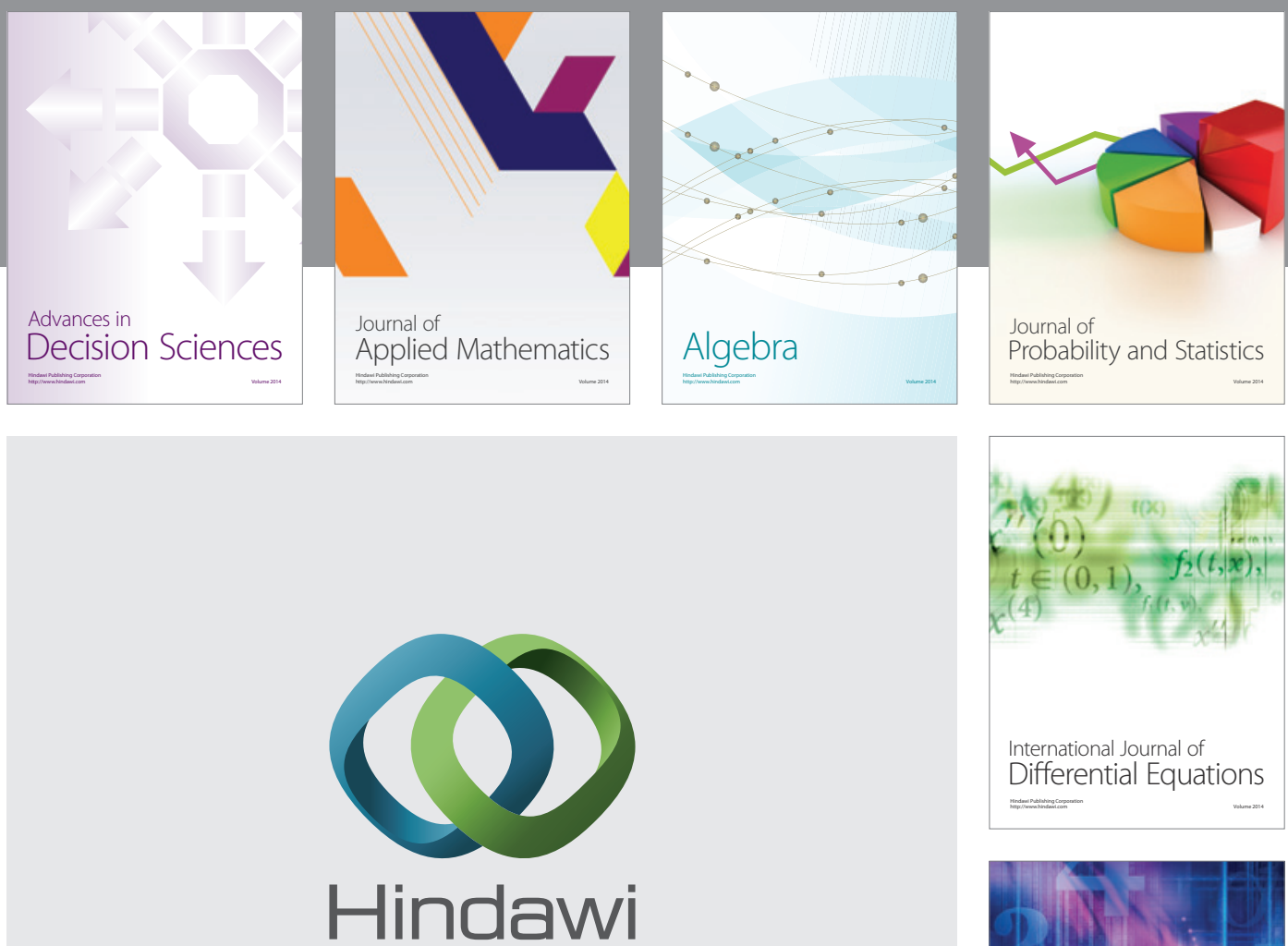

Submit your manuscripts at http://www.hindawi.com
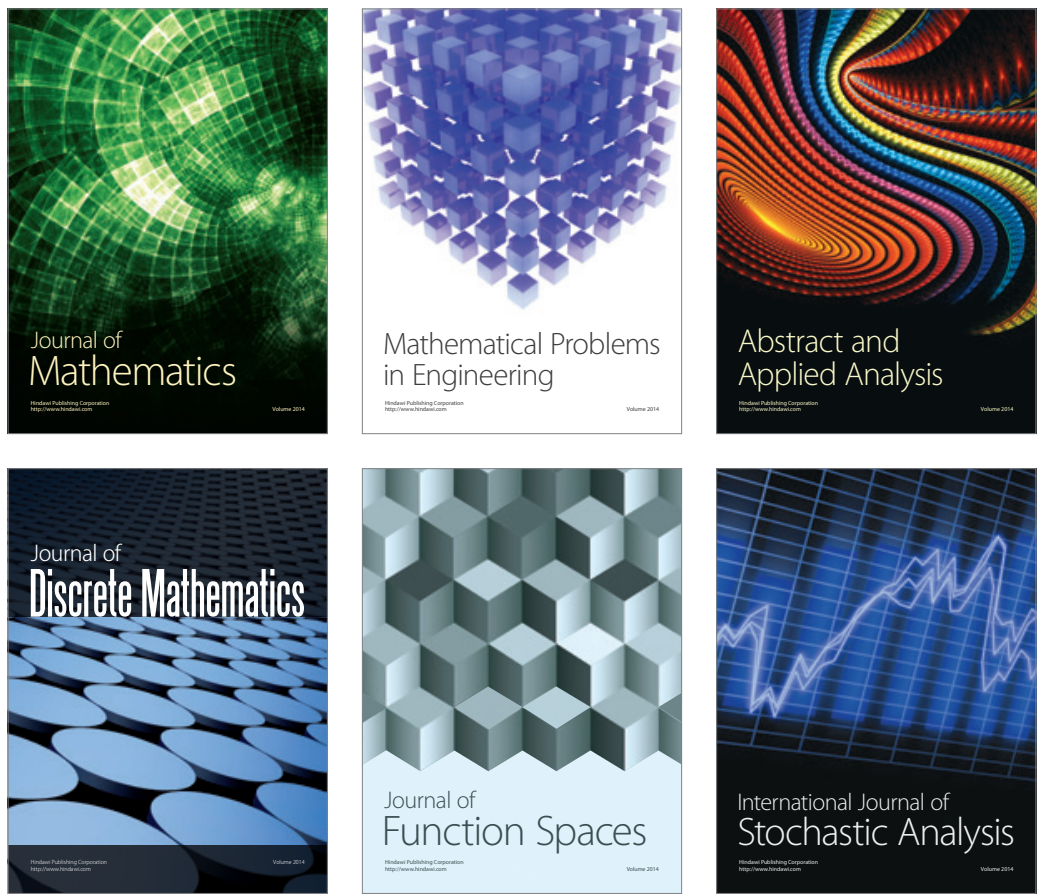

Journal of

Function Spaces

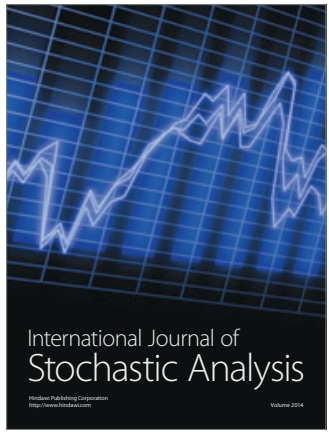

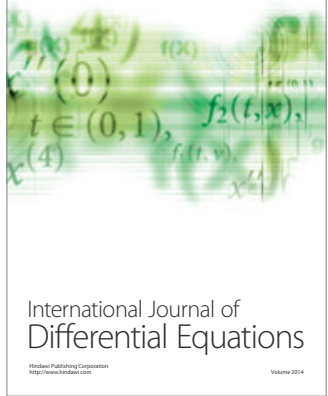
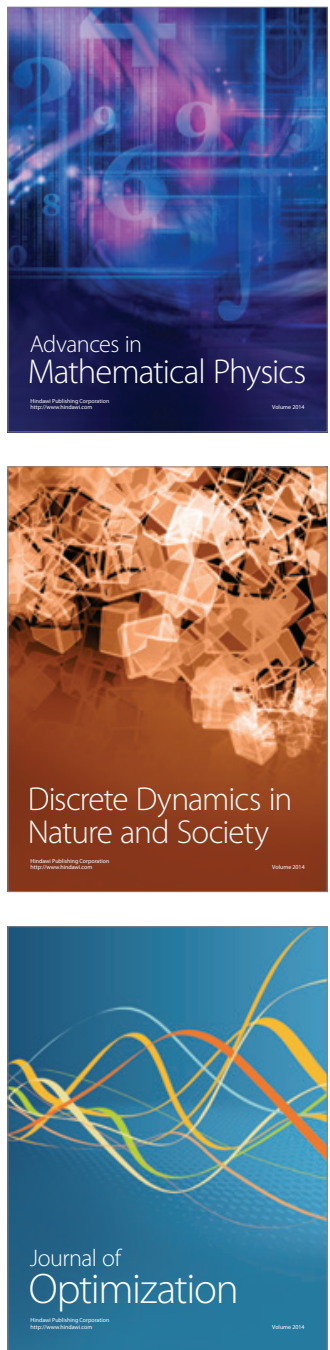